\title{
New mechanism of tolerance induction in cancer
}

The induction of T-cell tolerance is an important mechanism of tumour escape. Previous studies had shown a role for antigen-presenting cells in tumour-associated T-cell tolerance but the mechanisms involved were unknown. Now, Gabrilovich and colleagues show that myeloid-derived suppressor cells (MDSCs) induce $\mathrm{CD}^{+} \mathrm{T}$-cell tolerance by blocking

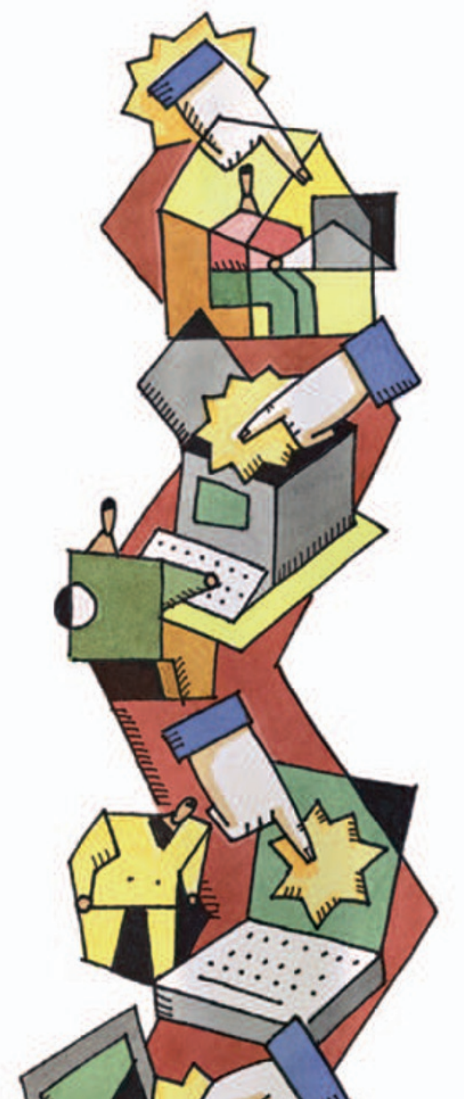

peptide-MHC (major histocompatibility complex)-TCR (T-cell receptor) binding through the nitration of the TCR-CD8 complex.

MDSCs are a group of immature $\mathrm{GR} 1^{+} \mathrm{CD} 11 \mathrm{~b}^{+}$cells (which include precursors of macrophages, granulocytes, dendritic cells and myeloid cells), that are produced in response to various tumour-derived cytokines. These cells have been shown previously to induce tumour-associated antigen-specific $\mathrm{CD}^{+} \mathrm{T}$-cell tolerance. To examine the mechanism involved, the authors used an experimental mouse model that allowed them to study the induction of T-cell tolerance in the absence of additional tumour-dependent effects. They found that antigen-specific $\mathrm{CD}^{+}$ $T$ cells from mice that had received MDSCs from EL-4-tumour-bearing mice did not bind as efficiently to specific soluble peptide-MHC complexes as cells from mice that did not receive MDSCs, suggesting that there was a defect in peptide-MHC-TCR binding.

One way of impeding the function of proteins (and therefore disrupting binding) is through the nitration of tyrosine residues. Examination of nitrotyrosine levels in $\mathrm{CD}^{+} \mathrm{T}$ cells incubated with MDSCs and peptide showed that MDSCs can induce nitrotyrosine formation in these cells in both TCR $\beta$ and CD8 molecules. These data and further molecular modelling suggested that nitration of the TCR-CD8 complex might affect its ability to interact and respond to the peptide-MHC complex, without affecting the ability of the $\mathrm{T}$ cell to respond to non-specific stimuli.

But how do MDSCs induce nitrotyrosine formation? Peroxynitrite can modify proteins through nitration of various amino acids, and MDSCs have been shown to produce high levels of peroxynitrite, as well as reactive oxygen species (ROS), which contribute to the generation of peroxynitrite. Inhibition of peroxynitrite by uric acid in MDSCs blocked the induction of antigen-specific $\mathrm{CD} 8^{+}$ T-cell tolerance. Finally, addition of uric acid to a cancer vaccine greatly enhanced the efficacy of the vaccine by substantially delaying tumour growth.

So, the data show that MDSCs, through the production of peroxynitrite and ROS and the nitration of the TCR-CD8 complex, induce $\mathrm{CD}^{+} \mathrm{T}$-cell tolerance by disrupting peptide-MHC-TCR binding. Preventing peroxynitrite generation, and thereby the induction of T-cell tolerance, could represent a potential therapeutic option to enhance the efficacy of cancer immunotherapy.

ORIGINAL RESEARCH PAPER Nagaraj, S. et al. Altered recognition of antigen is a mechanism of $\mathrm{CD} 8^{+} \mathrm{T}$ cell tolerance in cancer. Nature Med. 13 , 828-835 (2007) 